\title{
Mirror Mirror on the Wall: The Patients' View
}

\author{
Martha L. Deed
}

Published online: 12 June 2013

(C) Society for Imaging Informatics in Medicine 2013

There is nothing we can't handle here at the Gates Vascular Institute.

L. Nelson "Nick" Hopkins, MD, in a commercial for The Stroke Center at the Gates Vascular Institute, Buffalo, NY

\section{Introduction}

If you were a kid in the 1940s and 1950s and your parents really loved you, they took you to Mr. Glynn's shoe store where you could stick your feet in a box and examine them in the ghostly light of a flouroscope. And if you were a good customer (quiet, cooperative, and calm), Mr. Glynn would let you stop off on the way home from Liberty Street School for an extra peek.

This initial experience with a radiologist's toolbox highlights many of the issues which consumers still assume:

If it doesn't hurt, it's safe

If it's used routinely and often, it's safe

All X-ray, CT, and MRI machines are created equal

All machines are accessible to everyone

Turns out, those fluoroscopes in shoe stores were not such a good idea [1]. And subsequent events have shown that the rest of those assumptions are also questionable.

This paper, written from the patient perspective, outlines both the promise of imaging for well-informed patients as well as documenting the dangers that arise when patients receive

Author, The Last Collaboration, initially published at www.furtherfield.org/friendsofspork, available in book form at Amazon.com.

M. L. Deed $(\square)$

1037 Sweeney Street, North Tonawanda, NY 14120, USA

e-mail: mldeed@verizon.net

M. L. Deed

Access to Patient Information Program, NYS Department of Health, Albany, NY, USA

M. L. Deed

Consumer Union's Safe Patient Project, Yonkers, NY, USA incomplete, biased, or inaccurate reports of radiologic findings, or when adequate technology is not available. Cases from one community demonstrate how the public learns and forms opinions about radiology facilities within their own communities.

Suggestions on more efficient use of imaging reports and enhanced patient access to imaging reports are offered in the concluding section of the paper. Recommendations to increase public awareness of local imaging facilities are included.

\section{What the Patients and the Public Know}

\section{Differential Diagnosis: Case 1}

The 35-year-old woman was morbidly obese from mismanaged steroid treatment of an autoimmune disease, severe Secondary Cushing's Disease, and osteoporosis. She fired her first pain specialist when he appeared drunk for her appointment. She refused her nurse practitioner's referral to the second, because an internet search revealed lawsuits and a department of health sanction. Now the NP made references to "drugseeking behavior" when they spoke.

The woman traveled 75 miles from home to meet the new pain specialist.

The new doctor said, "I am sending you for X-rays. You need to have them before you leave the hospital today." $\mathrm{X}$-rays revealed multiple vertebral fractures. Now the possibly drug-seeking patient became the stoic, heroic patient. Her pain medications were changed, treatment goals were set (a 3-point reduction on the 10 point pain scale) and discussions focused on her current functioning and what she could do with some relief.

Within a month, the patient was spending more time out of bed. An artist, she began creating new projects and getting them published.

Sometimes what looks like non-compliance, drug-seeking behavior, or doctor shopping may be the result of poor medical care, not patient dysfunction. The new doctor was the first 
pain doctor to touch this patient. Differentiating emotional and physical causes of pain may be difficult [2]. But it is necessary for ensuring optimal mental health and physical care.

\section{Public Awareness of Local Radiology Services: Case 2}

Two years before out-of-town x-rays revealed the cause of this woman's pain, the medical school in her home community had disbanded its radiology residency program [3] due to an inability to meet accreditation standards [4].

Unhappiness among Kaleida's radiologists, for instance, stemmed partly from the hospital's outdated equipment [5].

Doctors who could were leaving town in large numbers [6]. In 1998, Buffalo's Women and Children's Hospital had 19 pediatric radiologists. Only six remained in 2001, and they resigned, citing poor working conditions and poor pay [7]. A 2011 survey noted that Western NY was experiencing the most serious shortages in ER services statewide. Ninety-one percent of respondents in WNY reported their emergency rooms were not covered by certain specialties at all times and $82 \%$ said they had reduced services because of problems finding some specialists [8].

University at Buffalo Professor Bruce Jackson writes: [9]

[A] rich guy [went for] emergency treatment because . . . of inexplicable painful and debilitating symptoms.

[He] was quickly interviewed, $x$-rayed, poked and prodded, and then he was parked in a room after some overworked resident decided he was just a senile alcoholic in booze withdrawal.

One of [the patient's] physician friends ....went to visit him ... [T] he friend knew [he] wasn't an alcoholic. [W] hat they were doing to him was making everything worse. . . [The] friend called a senior hospital official. The senior official came in and asked for a report on the x-rays. It turned out no one had read the x-rays because ... no radiologist worked in the hospital over the weekend. Once the bigshots got involved, they found a radiologist [who] said [the patient] had a collapsed lung and was hemorrhaging ... These are all things they would have known 30 min after he'd been admitted, had anyone looked at the $\mathrm{x}$-rays ...

... if a rich guy ... can be nearly killed by the system that's supposed to be saving him, nobody is safe. . .

He concludes:

Not one physician I told about Jerry's near-death experience was surprised. Every physician I told about it told me his or her own atrocity story. . .

One surgeon said, "When people used to say to me, 'I've got to have this or that procedure, where should I go?' I'd tell them, 'Go to General or go to Millard or whatever.' Now I tell them, 'Go to Cleveland, go to Boston, go any place but Buffalo.' " [9]

Radiologists' Place in Medical Culture: Case 3

A 51 year-old man with a history of long-standing rheumatoid arthritis treated with steroids and a history of colon cancer, reports to a local emergency department on February 1,2012. His symptoms include severe lower back pain and difficulty passing either urine or feces. Lumbosacral $\mathrm{x}$ rays indicate "No plain film abnormalities of the lumbar spine." Chest x-ray is also normal. No further studies are recommended, and the man is discharged from the ER with no explanation for his pain other than arthritis.

Two weeks later, the man's primary care physician refers him for a lumbosacral MRI for "Severe low back pain and lumbar radiculopathy." Imaging is obtained from L1-2 to L5-S1. The scan revealed probable muscle spasm and minor arthritis. No further studies are recommended by the radiologist.

The man's pain increases and his excretory functions worsen.

On April 29, 2012, the man reports to another hospital for a CT of abdomen and pelvis. "There is an eccentric mass [in] the rectum measuring approximately $6 \mathrm{~cm}$ image 62. This extends to the urogenital diaphragm and there are a few shotty lymph nodes at the pelvic inlet in the retroperitoneum." Impression: "Rectal mass concerning for carcinoma."

Further imaging and colonoscopy follows and treatment is instituted for a large malignant colon cancer.

The tumor was inoperable. Treatment was initiated in early June 2012. At this writing, the outcome is not known.

This case raises questions about whether the flaws in communications among physicians may endanger patients [10]. Unaware of possible gaps, patients are not educated to ask questions that might lead to further exploration. This is especially true when the patient receives good news.

Diagnostic Imaging in Treatment Planning and Patient Access to Radiological Reports: Case 4

A man experiences sudden double vision and blurred vision in one eye. He notices that this eye is bulging. He reports to a local emergency department. CT scan reveals a "focal left orbital abnormality [described under findings]. This most likely represents hemangioma. Differential diagnosis would also include orbital pseudotumor, lymphoma atypical infection as well as metastatic disease. Given clinical history, focal hemorrhage is not excluded. Followup MRI with and without gadolinium would be helpful for further evaluation." 
As a result of further testing, the man underwent left orbitotomy. In his post-surgery report, the surgeon wrote:

There is a large dilated vessel just posterior to the globe and had the appearance of a hemangioma. A suture was placed through this with drainage and the vessel was then dissected back further. It did not appear to be the reason for the orbital tumor.

No tumor was found. Multiple biopsies were negative for malignancy. Further imaging found no evidence of tumor.

There are at least two problems for the patient. The first is this man's pre-surgery vision was 20/25 with corrective lenses. The surgery left him blind in that eye. He can barely discern shadows. The second is that post-surgery, he has yet to see imaging test results that along with other records he has not been able to obtain might explain how the decision was made to recommend this surgical procedure to him.

\section{Access to Diagnostic Imaging: Case 5}

"There's nothing we can't handle here," Neurosurgery Chief Dr. L. Nelson "Nick" Hopkins, MD, FACS says in a cheerfully lit commercial [11] as he drives down a Buffalo street announcing the arrival of the new tertiary stroke center serving the six Western NY Counties. But it is not true. And further, what can be handled may take precious extra time.

Time is brain. Saving even 15 minutes in the lead time to diagnosis can make a significant difference in clinical decision-making and guide patient treatment, which impacts outcomes [12].

In 2009-2010, the number of obese adults in the USA was estimated at 78 million [13]. Of these, 3.8 million adults $(4.9 \%$ of all obese adults) weighed more than 300 lbs [14]. In 20092010, there were an estimated 308,300 obese adults living in the six county stroke center catchement area [15]. Assuming that $4.9 \%$ of obese adults in WNY weigh more than $300 \mathrm{lbs}$., approximately 15,100 people in WNY carry more than $300 \mathrm{lbs}$.

High rates of stroke and heart attack are associated with overweight and obese people [16]. WNY has relatively high rates of stroke [17].

Gates Vascular Institute refers patients who weigh more than $300 \mathrm{lbs}$ to other facilities for their MRI studies [18].

The need for immediate access to MRIs and other technologies with weight specifications is not limited to stroke patients [19]. In WNY, in-hospital MRI is unavailable for patients weighing more than $300 \mathrm{lbs}$. When MRI is required, patients must be transferred to a freestanding imaging facility and scheduled during normal weekday working hours. Arrangements are so complex that these patients may wait days for their studies if they are able to obtain them at all.
The 36 year-old morbidly-obese woman is in the ICU recovering from $\mathrm{H} 1 \mathrm{~N} 1$ in 2009 . On her 15th hospital day, shortly after a rough linen change during which she felt something snap in her back and her pain levels increased dramatically, she becomes paralyzed from the chest down in the space of a few hours. Although she has had CT scans at this hospital previously and at this weight, and although her family advocate produces the test results to prove this point, the advocate and hospital staff argue for nine days over whether a study can be done. The CT indicates a "lytic lesion" at the approximate point where the paralysis begins. The radiologist recommends scintigraphy, and staff informs the patient advocate that an MRI is needed but that no machine in the system can accommodate her.

The patient herself apparently that further imaging studies are urgent. Intubated, she writes:

If it is compression, is there any treatment anyway?

But is time an issue with establishing diagnosis?

So maybe it's OK to wait and to work toward getting me extubated [20].

Her doctors make repeated notations in the patient's medical record, e.g.:

Neuro - ideally need a MRI of thoracic lumbar spine paralysis unchanged, was incontinent of stool - transverse myelitis vs cord compression not likely GBS although IVIG can take awhile to work. Neuro consult appr'd. We need imaging but to make that possible we need to wean off ventilator [20].

Days later, a second neurologist personally makes arrangements for testing at his center, three miles from the hospital on an MRI table rated for $650 \mathrm{lbs}$. But the first available appointment is in 10 days nearly three weeks after the onset of sudden paralysis. The patient dies before she gets her MRI.

Autopsy reveals a spinal infection had severed her spinal cord [20].

The woman's treating rheumatologist, who was not part of the in-hospital treatment team, told the family that the woman was not terminally ill and that her life most likely would have been saved had she obtained prompt diagnostic imaging, been aggressively treated with antibiotics, and the infected bone had been removed from her spine.

Obesity did not kill this patient. In a contemporary culture where it is acceptable for physicians to refuse to treat patients based on their weight [21], the lack of urgency in arranging appropriate studies for the suddenly paralyzed woman suggests that bias killed this patient [22]. Time was crucial. Testing was available three miles from her hospital bed. Neither the patient nor the family advocate were given any indication that the recommendation for an MRI was urgent. 
This situation did not occur due to a lapse on the radiologist's part. His recommendation was not appropriately pursued.

\section{Discussion and Recommendations}

The cases and circumstances outlined above were selected to demonstrate what patients are likely to know about their local radiology services and to illustrate how communication barriers may impair or enhance patient outcomes.

Generally, patients do not speak directly with radiologists who interpret their studies. When the referring doctor accurately comprehends the differential diagnoses that may explain the patient's symptoms and medical history, writes appropriate orders for imaging, obtains reports promptly, accurately relates the radiologist's impressions, discusses the results and treatment plans with the patient, and does so in a timely fashion, the patient is likely to receive maximum benefit from the radiologist's expertise.

If the referring doctor provides a copy of the report to the patient, the patient benefits further, because the patient has documentation both of the results and also of what type of imaging was performed and where it was performed.

Patients are often unaware of access to radiologists and imaging facilities in their own communities. As case 2 illustrates, even well-educated (and well-heeled) patients may not be aware that services are lacking.

- Especially, in hospital emergency departments, patients should be told whether a radiologist is on site.

- If the general public knows that gaps in radiology equipment and staffing exist, pressure can be exerted to address these issues. Openness can benefit both the radiologists and patients.

If the referring doctor's order does not accurately reflect the patient's complaints (case 3 ), the diagnostic study results may not be relevant.

- Prior to performing diagnostic imaging, referrals should be checked against the patient's medical history to confirm that the most relevant studies have been selected.

Case 4 addresses communication barriers between patients and radiologists. Hospitals including Cleveland Clinic [23] and some physicians now provide medical reports to patients as soon as they are generated. In the childhood game of "Telephone," messages become lost or distorted as they are passed from one player to the next around the circle. Safe to say the same can happen within the medical culture.

- Radiologists can require referring doctors to permit direct transmission of reports to patients at the same time that they are sent to the referral sources.
Case 5 raises similar issues to case 4 , since in both cases, radiologic studies may not have been put to optimal use. Case 5 is really an access issue that directly involves radiologists. Both in the hospital and in the community-at-large, access issues are paramount. Given that extremely obese people are at enhanced risk for conditions such as cardiovascular events which require rapid diagnosis for optimal outcomes, lack of access may create ethical issues for radiologists [24].

There has been no media coverage of the weight limits of MRI and CT scanners in Western New York, so it is doubtful that the public is aware of the limitations.

- Raiology equipment weight limitations at all local hospitals should be made publicly available.

- Protocols to transport of patients 24/7 to facilities where studies can be performed should be developed and made public.

- At least one local inpatient hospital should be equipped to make a full range of imaging studies available $24 / 7$ for patients who weigh more than $300 \mathrm{lbs}$. These patients might still experience significant delays due to authorizations and transport arrangements, but their survival rates might well improve.

- Radiologists as well as the medical community as a whole should make sure that the increased health risks of obesity are not compounded by bias against fat people which leaves doctors willing to offer them less than optimal care. It is an ethical problem that the level of radiological care in this community is less for an estimated 15,100 people than for the rest of the community.

Finally, it seems quite possible that the issues raised in this paper by someone who is not a radiologist may already be well-known in the radiology community and are being actively addressed. The issues raised here are offered from a patient's perspective in the hope that radiologists can strengthen their connections with the patients they treat and the doctors who refer to them.

\section{References}

1. Smithsonian Magazine Blog. http://blogs.smithsonianmag.com/ design/2012/04/better-feet-through-radiation-remembering-theera-of-the-fluoroscope/. Accessed 28 August 2012

2. Disorbio JM, Bruns D, Barolat G: Assessment and treatment of chronic pain. Practical Pain Management 2, 2006

3. Accreditation Council of Graduate Medical Education. List of withdrawn programs: Academic Year 2006-2007. http://www.acgme.org/ adspublicreports/withdrawn_prog_list.asp. Accessed 30 July 2012

4. Franczyk A: UB alters 2 residency hospital programs. Business First September 20, 2006. http://www.bizjournals.com/buffalo/stories/ 2005/09/18/daily30.html. Accessed 30 July 2012

5. Buffalo News-Medical Exodus. www.buffnews.com 31 Mar 2002 [cached] at http://www.zoominfo.com/\#!search/profile/person?personId= 
150960344\&targetid=profile. Accessed 28 July 2012. Note that Dr. Mogavero is a current board member of the hospital he criticized in this newspaper story

6. Franczyk A: Sick system: exodus of doctors threatens health care in WNY. Business First. http://www.bizjournals.com/buffalo/stories/ 2001/08/13/story3.html. Accessed 30 July 2012

7. Claiming Overwork, Hospital's Radiologists Resign AP August 3, 2001.

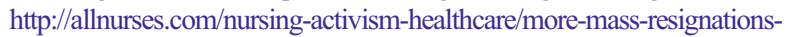
8926.html. Accessed 19 August 2012

8. Davis H: Shortage of doctors in state worst here $/ 91 \%$ of hospital Ers lacking specialists. http://www.buffalonews.com/city/article312141.ece January 14, 2011. Accessed August 18, 2012

9. Jackson B: Hospitals at the edge. Part 1: Buffalo's most dangerous neighborhood. http://www.ascu.buffalo.edu/ bjackson/hospitals1.html. Accessed 18 August 182012

10. Health Grades. Patient safety and satisfaction: The State of American Hospitals. https://www.cpmhealthgrades.com/CPM/ assets/File/HealthGradesPatientSafetySatisfactionReport2012.pdf, p.3. Accessed 28 August 2012

11. Kaleida Health. http://www.kaleidahealth.org/gvi/. Accessed 19 August 2012

12. Michael Miller MD quoted in Health Imaging \& IT. http:// www.healthimaging.com/index.php?option $=$ com_articles\&view $=$ article\&id=17431:volume-ct-the-key-to-a-superior-stroke-protocol. 2009. Accessed 19 August 2012

13. Ogden CL, Carroll MD, Kit BK, Flegel KM: Prevalence of obesity in the United States, 2009-2010. NCHS Data Brief, No 82, CDC, January 2012

14. National Council on Strength and Fitness. http://www.ncsf.org/ enew/articles/articles-obesityawarenessmonth.aspx. Accessed 20 August 2012
15. County-Specific Report-Expanded BRFSS July 2008—June 2009. http://www.health.ny.gov.statistics/brfss/expanded/2009/county/. Accessed 29 August 2012

16. Hubert HB, Feinleib M, McNamara PM, Castelli WP: Obesity as an independent risk factor for cardiovascular disease: a 26-year followup of participants in the Framingham Heart Study. Circulation 67:968-977, 1983. doi:10.1161/01.CIR.67.5.968

17. Research Center for Stroke \& Heart Disease. http://www.strokeheart.org/ stats.html. Accessed 29 August 2012

18. Kaleida Health. http://www.kaleidahealth.org/Stroke/diagnostics/. Accessed 19 August 2012. Author spoke to staff in MRI office to confirm that patients who weigh more than $300 \mathrm{lbs}$ are transferred to outpatient imaging center August 20, 2012

19. Siemens Medical Solutions: MRI Hot Topics: Obesity and MR Imaging. Order No. A9119-6218-1C-4A00. http://www.medical.siemens.com/ siemens/en_US/rg_marcom_FBAs/files/brochures/cs_diagnostic/ obesity_HT__v12.pdf. Accessed 19 August 2012

20. Deed M: The last collaboration. Furtherfield, Buffalo, 2012. 136

21. Campos P: Anti-obesity: The new homophobia? Salon. http:// www.salon.com/2012/08/28/anti_obesity_the_new_homophobia/. Accessed 31 August 2012

22. Western NY Physician: Safely Caring for Obese Patients in the Office Practice Setting. http://westernnyphysician.com/PDF/January-February2011.pdf. 20. Accessed 29 August 2012

23. Szilagyi S: The Patient Experience. http://cchealth.clevelandclinic.org/ cover-story/patient-experience. Accessed August 22, 2012

24. American College of Radiology. http://www.ama-assn.org/ama/pub/ physician-resources/medical-ethics/about-ethics-group/ethics-resource-center/educational-resources/federation-repository-ethicsdocuments-online/american-college-radiology.page. Section 2: 7. Accessed 18 August 2012 